Cahiers $d u$ MONDE RUSSE

\section{Cahiers du monde russe}

Russie - Empire russe - Union soviétique et États indépendants

43/2-3 | 2002

Contacts intellectuels, réseaux, relations internationales

\title{
La Révolution française : de l'historiographie soviétique à l'historiographie russe, « changement de jalons »
}

\author{
Alexandre V. Tchoudinov
}

\section{CpenEdition}

Journals

Édition électronique

URL : https://journals.openedition.org/monderusse/8509

DOI : 10.4000/monderusse.8509

ISSN : $1777-5388$

Éditeur

Éditions de l'EHESS

Édition imprimée

Date de publication : 1 avril 2002

Pagination : 449-462

ISBN : 2-7132-1781-4

ISSN : $1252-6576$

Référence électronique

Alexandre V. Tchoudinov, "La Révolution française : de l'historiographie soviétique à l'historiographie russe, "changement de jalons » », Cahiers du monde russe [En ligne], 43/2-3 | 2002, mis en ligne le 01 janvier 2007, consulté le 03 septembre 2022. URL : http://journals.openedition.org/monderusse/8509 ; DOI : https://doi.org/10.4000/monderusse.8509 
chercher : repérer : avancer

Cet article est disponible en ligne à l'adresse :

http://www.cairn.info/article.php?ID REVUE=CMR\&ID NUMPUBLIE=CMR 432\&ID ARTICLE=CMR 4320449

\title{
La Révolution française : de l' historiographie soviétique à l' historiographie russe, « changement de jalons »
}

\author{
par Alexandre V. TCHOUDINOV
}

\section{Editions de l'EHESS | Cahiers du monde russe}

\author{
2002/2-3 - Vol 43 \\ ISSN 1252-6576 | ISBN 2713217814 | pages 449 à 462
}

Pour citer cet article :

-V. TCHOUDINOV A., La Révolution française : de I'historiographie soviétique à l' historiographie russe, " changement de jalons ", Cahiers du monde russe 2002/ 2-3, Vol 43, p. 449-462.

Distribution électronique Cairn pour les Editions de l'EHESS.

(C) Editions de l'EHESS. Tous droits réservés pour tous pays.

La reproduction ou représentation de cet article, notamment par photocopie, n'est autorisée que dans les limites des conditions générales d'utilisation du site ou, le cas échéant, des conditions générales de la licence souscrite par votre établissement. Toute autre reproduction ou représentation, en tout ou partie, sous quelque forme et de quelque manière que ce soit, est interdite sauf accord préalable et écrit de l'éditeur, en dehors des cas prévus par la législation en vigueur en France. Il est précisé que son stockage dans une base de données est également interdit. 


\section{LA RÉVOLUTION FRANÇAISE : DE L'HISTORIOGRAPHIE SOVIÉTIQUE À L'HISTORIOGRAPHIE RUSSE, «CHANGEMENT DE JALONS »}

L'étude de la Révolution française dans les universités et les académies russes débute dans les années 70 du XIX ${ }^{\mathrm{e}}$ siècle et s'impose immédiatement comme l'une des orientations prioritaires de l'historiographie nationale. On lui accorde alors un sens tout particulier, voire prophétique : la société russe scrute l'histoire de la France du XVIII ${ }^{\mathrm{e}}$ siècle comme si cette dernière lui tendait un miroir où déchiffrer l'avenir. Ce sont bel et bien les réalités de l'empire russe qui, dès le début, définissent le choix des thèmes de recherche ${ }^{1}$. Ainsi, l'actualité brûlante de la question agraire dans la Russie d'avant la révolution suscite-t-elle un très grand intérêt pour les problèmes agraires auxquels la Révolution française a été confrontée, et elle est à l'origine des travaux classiques de la fameuse «école russe » représentée par Nikolaj Kareev, Ivan Lučickij, Maksim Kovalevskij.

À l'époque soviétique, l'influence du contexte politique sur l'orientation de l'étude de la Révolution française se renforce encore. La direction du PCUS lui accorde une signification politique majeure, qui confine à la sacralisation ${ }^{2}$. Seule, la révolution d'Octobre se voit attribuer plus d'importance. Dans les années 19201930, l'historiographie soviétique, placée sous le contrôle idéologique strict du parti et de l'État, élabore une interprétation canonique de la Révolution française, exposée sous sa forme définitive dans un ouvrage fondamental publié en $1941^{3}$. Dans un premier temps, il était prévu de le publier pour le 150 e anniversaire de la

1. Voir Dmitry Shlapentokh, The French Revolution in Russian intellectual life. 1865-1905, Wesport (Conn.) - Londres, 1996 ; id., The French Revolution and Russian anti-democratic tradition. A case of false consciousness, New Brunswick - Londres, 1997.

2. T. Kondratieva, Bolcheviks et Jacobins : itinéraire des analogies, Paris, 1989.

3. Francuzskaja buržuaznaja revoljucija, 1789-1794 (La Révolution bourgeoise française, 1789-1794), Moscou - Leningrad, 1941. 
Révolution française, mais la répression qui s'abat en 1937-1938 sur de nombreux membres du collectif de rédaction, et au premier chef sur son responsable, l'académicien Nikolaj Lukin, cousin de Nikolaj Buharin, entraîne le retard constaté. Néanmoins c'est à « l'école de Lukin » que revient le principal mérite de l'élaboration de cette interprétation canonique qui va dominer la littérature scientifique soviétique jusque dans la première moitié des années 1980.

Le « changement de jalons » est à mettre en relation avec la commémoration du bicentenaire de la Révolution française. À dire vrai, ce processus ne relève pas tant du renouveau d'intérêt que suscite chaque commémoration, même si, reconnaissons-le, les efforts des chercheurs en ont été fortement stimulés ; on le doit plus encore aux développements logiques de la science elle-même, ainsi qu'aux changements induits par la perestroika dans la société et dans le domaine politique. La pression idéologique permanente du PCUS et de l'État à laquelle étaient soumis les historiens soviétiques spécialistes de cette discipline va s'affaiblissant à partir du milieu des années 80 pour finir par disparaître, ce qui rend possible une recherche indépendante, des expériences sur le plan méthodologique et une approche constructive des acquis de la recherche à l'étranger.

Un autre facteur influe largement sur le développement de ce secteur de l'historiographie au cours de ces quinze-vingt dernières années : il s'agit de la relève des générations. Dans les années 50-70, ses têtes de file reconnues étaient les disciples de Lukin, Albert Manfred (1906-1976) et Viktor Dalin (1902-1985), qui représentaient la génération des « romantiques de la Révolution », venus à la science historique peu après 1917. À leur mort, le relais est pris par Anatolij Ado (1928-1995) et Gennadij Kučerenko (1932-1997), disciples de l'historien et philosophe bien connu Boris Poršnev, dont l'interprétation peu orthodoxe du marxisme avait plus d'une fois suscité le mécontentement des organes du parti. Ayant tous deux achevé leur formation au moment du «dégel », ils ont eu la possibilité de faire des recherches en France dès les années 60. Éloignés du marxisme-léninisme militant de leurs prédécesseurs et bien plus ouverts qu'eux aux approches de l'historiographie contemporaine mondiale, ils se sont efforcés d'inculquer ces qualités à leurs élèves. Aussi les changements radicaux qui se sont produits au cours de ces deux dernières décennies dans l'historiographie russe de la Révolution française sont-ils largement liés à l'activité de ces chercheurs et des écoles qu'ils ont fondées ${ }^{4}$.

Le domaine dans lequel le changement de jalons se fait sans doute le plus sentir est la modification de l'attitude des chercheurs russes à l'égard des orientations non marxistes de l'historiographie occidentale qui suscitaient chez les historiens soviétiques une réaction uniformément négative. Ainsi, quand ces derniers s'engagent, au milieu des années 70, dans la fameuse dispute lancée à l'Ouest entre les partisans d'une lecture «classique» et ceux d'une lecture

4. Sur les vingt thèses de troisième cycle soutenues de 1986 à 1997 sur l'histoire de la Révolution française, onze venaient des disciples d'Ado et de Kučerenko (respectivement, six et cinq) ; de même pour trois thèses d'État sur quatre (respectivement, deux et une). 
« révisionniste » de la Révolution, ils font tout pour donner à la polémique un ton plus âpre que jamais, traitant tout simplement les « révisionnistes » d'ennemis du progrès et de la démocratie et déniant tout fondement scientifique à la querelle. En 1976, Manfred écrit dans la revue Kommunist, organe théorique central du CC du PCUS : «Les flèches décochées contre la Révolution française visent plus loin ; elles sont destinées à la Grande Révolution socialiste d'Octobre, à la puissante URSS, au système socialiste mondial, au mouvement ouvrier, à celui de libération nationale, à toutes les forces démocratiques, progressistes auxquelles le devenir de l'humanité est lié. ${ }^{5}$ Cet article, le dernier à avoir été publié du vivant de l'historien, résonne comme une sorte de testament politique du chef incontesté de ce courant de la science historique soviétique. En effet, tous les travaux consacrés à l'analyse de l'historiographie « révisionniste » publiés à la charnière des années 70-80, si ce n'est par la forme, du moins dans l'esprit, correspondent parfaitement à l'approche d'Albert Manfred. En dépit d'un respect tout extérieur des formes académiques, leurs auteurs poursuivent avant tout un objectif idéologique : démontrer l'inconsistance scientifique de tout écart vis-àvis de l'interprétation marxiste de la Révolution ${ }^{6}$.

Leur tâche est grandement simplifiée du fait que les travaux du courant « révisionniste » restent inaccessibles à de vastes milieux scientifiques en URSS : on ne peut les consulter que si l'on a accès à des réserves spéciales... Les disciples d'Ado m'ont raconté qu'ils devaient se tenir à l'écart des regards indiscrets pour lire le livre de François Furet, Penser la Révolution française, que leur maître avait rapporté de France. D'ailleurs, les articles de ce même Ado sont à l'époque pour les historiens soviétiques une importante source d'information sur les interprétations non marxistes de la Révolution française. Tout en polémiquant, comme ses collègues, avec les «révisionnistes», il s'efforce avant tout d'éclairer avec un maximum d'objectivité et de détails la position de ses contradicteurs et, dans la mesure du possible, de s'éloigner des aspects idéologiques de la controverse pour aborder la discussion de problèmes scientifiques concrets.

Bien entendu, il ne peut être alors question d'une reconnaissance publique de la réalité scientifique des questions en discussion. Cela ne va devenir possible qu'avec l'affaiblissement du contrôle idéologique au moment de la perestroïka. Dès 1986, procédant à l'analyse des derniers travaux des « révisionnistes », Ado déclare : « Il ne serait pas juste de considérer que ces travaux n'accordent pas d'attention à certains problèmes réellement complexes et importants de l'histoire de la France de

5. A. Z. Manfred, Velikaja francuzskaja revoljucija (La Grande Révolution française), Moscou, 1983, p. 419.

6. M. N. Sokolova, Sovremennaja francuzskaja istoriografija (L'historiographie française contemporaine), Moscou, 1979; Iu. N. Afanas'ev, Istorizm protiv eklektiki : francuzskaja istoričeskaja škola «Annalov» V sovremennoj buržuaznoj istoriografii (Histoire contre éclectisme : l'école historique française des «Annales» dans l'historiographie bourgeoise contemporaine), Moscou, 1980 ; V. M. Dalin, Istoriki Francii XIX-XX vV. (Les historiens de France $X I X-X X^{\mathrm{e}}$ siècles), Moscou, 1981. 
cette époque. $\gg^{7}$ De nouveaux pas sont franchis dans cette direction lors d'une table ronde à l'Institut d'histoire universelle de l'Académie des sciences, les 19 et 20 septembre 1988. Ce forum auquel prennent part pratiquement tous les spécialistes russes de la Révolution française en activité à ce moment-là aura une portée décisive en ce qui concerne le développement ultérieur des recherches dans ce domaine. Plus précisément, beaucoup d'intervenants y soulignent la nécessité, pour notre historiographie, d'assimiler les acquis de toutes les tendances de la science historique contemporaine, sans exclusive aucune ${ }^{8}$.

La dernière décennie marque un changement radical. Une série de monographies est publiée, qui analysent divers aspects des lectures « révisionnistes » et conservatrices de la Révolution française ${ }^{9}$. Les ouvrages des fondateurs de l'historiographie conservatrice, Edmund Burke et Joseph de Maistre, sont traduits en russe ; le livre de Furet, Penser la Révolution française, interdit par la censure soviétique, comme on l'a déjà dit, sort en 1998 à Saint-Pétersbourg. Tout cela concourt à un élargissement considérable de l'horizon méthodologique des spécialistes russes de la question. Cependant la réévaluation essentielle du rôle des orientations non marxistes à laquelle la science historique russe a procédé n'a pas eu pour effet de rejeter l'héritage scientifique de l'historiographie «classique », en particulier marxiste, dont relevaient les chercheurs soviétiques. Ainsi la publication récente d'un volume d'hommage à Viktor Dalin à l'occasion du 95e anniversaire de sa naissance, apparaît comme une preuve de respect pour la mémoire des prédécesseurs ${ }^{10}$.

Les chercheurs russes n'ont fait que se délester de la surcharge idéologique qui pesait sur l'étude de la Révolution française, ce qui leur permet, parallèlement à l'acquisition du pluralisme méthodologique, de résoudre des problèmes concrets en s'appuyant sur l'expérience positive de toutes les traditions historiographiques. Ce

7. A. Ado, «Velikaja francuzskaja revoljucija i ee sovremennye kritiki » (La Grande Révolution française et ses critiques contemporains), in Buržuaznye revoljucii XVII-XIX vV. v sovremennoj istoriografii (Les révolutions bourgeoises des XVII-XIX $X^{\mathrm{e}}$ siècles dans l'historiographie contemporaine), Moscou, 1986, p. 109.

8. Aktual'nye problemy izučenija istorii Velikoj francuzskoj revoljucii (Problèmes actuels de l'étude de l'histoire de la Grande Révolution française), Moscou, 1989.

9. S. F. Blumenau, «Revizionnistskoe » napravlenie v sovremennoj francuzskoj istoriografii Velikoj buržuaznoj francuzskoj revoljucii konca XVIII v. (Le courant « révisionniste » dans l'historiographie française contemporaine de la Grande Révolution bourgeoise française de la fin du XVIII siècle), Brjansk, 1992 ; id., Spory o revoljucii vo francuzskoj istoričeskoj nauke vtoroj poloviny 60-70yh godov (Les débats autour de la Révolution française dans la science historique française de la seconde moitié des annnées 60 et des années 70), Brjansk, 1994 ; id., Ot social'no-ekonomičeskoj istorii k problematike massovogo soznanija : Francuzskaja istoriografija revoljucii konca XVIII v. (1945-1993) (De l'histoire sociale et économique à la problématique de la mentalité des masses : L'historiographie française de la révolution de la fin du XVIII siècle (1945-1993)), Brjansk, 1995 ; A. V. Cudinov, Razmyšlenija angličan o Francuzskoj revoliucii : E. Berk, D. Makintoš, U. Godvin (Les réflexions des Anglais sur la Révolution française : E. Burke, J. Mackintosh, W. Godwin), Moscou, 1996.

10. A. V. Čudinov, ed., Istoričeskie etjudy o Francuzskoij revoljucii. Pamjati V. M. Dalina (1902-1985) (Etudes historiques sur la Révolution française. Hommage à V. M. Dalin (19021985)), Moscou, 1998. 
faisant, les historiens russes recouvrent cet avantage qu'évoquait Alexandre Herzen au XIX ${ }^{\mathrm{e}}$ siècle :

« L'historien occidental est juge et partie à la fois ; les haines et les querelles de famille ne se sont pas tues en lui, il est l'homme de l'une des parties, sinon c'est un égoïste apathique ; il est trop impliqué dans la dernière page de l'histoire européenne pour ne pas se sentir directement concerné par la première et les suivantes. Il n'y a pas de position plus objective pour juger de l'histoire occidentale que la position du Russe. $»^{11}$

Une problématique élargie, l'application de méthodes nouvelles ont contribué à introduire dans l'historiographie russe des matériaux factuels considérables qu'on ne peut expliquer en restant dans le cadre de l'interprétation soviétique canonique de la Révolution française. Cette interprétation repose sur la théorie dite « marxiste-léniniste», en réalité stalinienne, des formations économiques et sociales qui, en général, était exprimée de la manière suivante : «la révolution bourgeoise » a détruit en France « le régime de l'absolutisme féodal », ouvrant la voie à « un développement sans entraves du capitalisme ». Quelque âpres qu'aient été les discussions qui ont mis aux prises les chercheurs soviétiques dans les années 20, puis dans les années 60-70, jamais il n'y eut de remise en cause du consensus sur ce schéma sociologique, sorte d'armature admise par l'historiographie soviétique pour traiter des événements dans la France de la fin du XVIIIe siècle.

Ainsi, la discussion sur le contenu de classe de la dictature jacobine qui opposa Albert Manfred et Viktor Dalin au professeur Vladimir Revunenkov, de Leningrad, dans les années 60-70, rencontra un large écho au sein de la société. Cette polémique se distingua par un ton d'une telle âpreté, d'une telle intolérance, que même les responsables du secteur scientifique du CC du PCUS durent par moments appeler les adversaires à plus de retenue. Et pourtant, en ce qui concerne le rôle historique de la Révolution française, les jugements des deux parties s'inscrivaient parfaitement dans le schéma «régime de l'absolutisme féodal-révolution bourgeoise-capitalisme ». Manfred écrivait : «La Révolution française a détruit jusqu'à ses fondements le régime de l'absolutisme féodal, a extirpé la féodalité, a chassé d'un gigantesque coup de balai toutes les vieilleries héritées du Moyen Âge et nettoyé le terrain pour un développement de nature capitaliste. » ${ }^{12}$ Revunenkov exprimait son opinion dans des termes à peu près identiques : «Cette révolution a balayé les structures dépassées héritées du Moyen Âge non seulement en France même, mais à bonne distance de ses frontières, donnant par là même une impulsion

11. A. I. Gercen, «Publičnye čtenija g. Granovskogo» (Les conférences publiques de M. Granovski), in id., Sočinenija v dvuh tomah (Herzen, Euvres en deux volumes), Moscou, 1985, t. 1, p. 203.

12. A. Z. Manfred, op. cit., p. 206. 
puissante à la formation d'un nouveau système socio-économique, le capitalisme et la démocratie bourgeoise [...]. »13

On ne trouvera pas aujourd'hui dans l'historiographie russe de la Révolution française l'exemple d'une polémique comparable dans sa brutalité à celle qui opposa A. Manfred et V1. Revunenkov, qu'elle soit engagée avec des historiens contemporains ou leurs prédécesseurs. Néanmoins les recherches effectuées en histoire sociale ces quinze-vingt dernières années, surtout par l'école d'Ado (Kučerenko et ses disciples se sont spécialisés pour l'essentiel dans l'histoire des idées), font peser le doute sur la valeur scientifique de ce fondement méthodologique appliqué à l'interprétation des événements révolutionnaires, fondement qui faisait l'objet d'un accord unanime auprès des historiens soviétiques.

Dans leurs travaux les chercheurs russes contemporains procèdent à la révision des représentations antérieures de l'Ancien Régime. On l'a dit, les auteurs soviétiques utilisaient, pour le définir, le concept de «régime féodalo-absolutiste». Manfred définissait de la façon suivante la cause première de la Révolution : «Le régime féodalo-absolutiste était dépassé, il ne correspondait plus au niveau socioéconomique du pays et, devenu une entrave au développement des forces productives, il faisait obstacle à leur croissance. ${ }^{14}$ Revunenkov utilisait ce même concept pour avancer une définition généralisatrice des structures économiques et politiques de la France prérévolutionnaire ${ }^{15}$.

À la lumière des études de ces quinze-vingt dernières années, le qualificatif de «féodal » pour définir les structures socio-économiques de l'Ancien Régime soulève chez les spécialistes russes de sérieux doutes. Il n'est pas sans intérêt de noter que, au début des années 80 , Viktor Dalin, qui préparait la réédition posthume de La Grande Révolution française de Manfred, a tenté de tempérer le jugement trop catégorique de son collègue et ami en cette matière. Dans l'édition de 1956, Manfred écrivait: "Globalement, dans l'agriculture française de la fin du XVIII ${ }^{\mathrm{e}}$ siècle dominaient encore des rapports anciens, moyenâgeux, de type féodal (souligné par moi-A.T.) sous leur forme la plus grossière, la plus sauvage [...]. » Dans cette phrase, Dalin remplace l'adjectif souligné par «semi-féodaux » ${ }^{16}$. Certes, un tel palliatif ne modifie pas radicalement la conception d'ensemble, mais cette tentative de correction témoigne qu'un représentant de l'historiographie soviétique aussi en vue que Dalin ne pouvait plus, dans les dernières années de sa vie, admettre sans réserve le caractère « féodal » de l'économie française à la veille de la Révolution.

13. V. G. Revunenkov, Očerki po istorii Velikoj francuzskoj revoljucii 1789-1814 gg. (Essais sur l'histoire de la Grande Révolution française, 1789-1814), 3e éd., Saint-Pétersbourg, 1996, p. 505 .

14. A. Z. Manfred, op. cit., p. 14.

15. V.G. Revunenkov, op. cit.,p. 44, 507.

16. A. Z. Manfred, op. cit., p. 17. 
Lors de la table ronde de 1988 mentionnée plus haut ${ }^{17}$, Ado évoque ce fait, peutêtre simplement curieux, néanmoins très caractéristique. Mais pour l'essentiel, sa communication est consacrée à la critique d'une « conception simplifiée, linéaire de la place de la Révolution française dans le processus de transition entre les différents stades de développement de la société, à savoir que si, en 1789, dominaient le régime féodal et la noblesse féodale, en 1799, vint le tour du capitalisme et de la bourgeoisie capitaliste », conception largement représentée dans l'historiographie soviétique. Une disciple d'Ado, L. Pimenova, se prononce encore plus nettement : «Qu'y avait-il de féodal dans la France du XVIII ${ }^{\mathrm{e}}$ siècle ? Quelque aspect de la vie que nous examinions, nulle part nous n'aurons un tableau univoque qui s'inscrira dans le cadre d'une définition par le concept de régime féodal. L'économie était multiforme, l'État et la société eux aussi se présentaient comme l'entrelacs complexe d'éléments hétérogènes. ${ }^{18}$

Et de fait, les résultats publiés dans les ouvrages d'Ado et de Pimenova juste avant la table ronde de 1988 remettaient en cause le bien-fondé des anciennes représentations concernant l'économie sous l'Ancien Régime, qui avaient si largement cours dans l'historiographie soviétique. Bien que le complexum feudale se soit maintenu jusqu'à la Révolution, et y ait même survécu dans certains secteurs, il ne jouait plus aucun rôle déterminant au XVIII ${ }^{\text {e }}$ siècle. Et Pimenova fait remarquer que, même dans les régions où l'économie présentait une structure archaïque, la part des droits seigneuriaux dépassait rarement $40 \%$ dans les revenus des seigneurs. Dans les régions économiquement avancées, elle était de beaucoup inférieure. La part essentielle de leurs revenus provenait d'une gestion capitaliste et semi-capitaliste de l'économie ${ }^{19}$. Plus encore, dans sa monographie, Ado soulignait que certains éléments du complexum feudale, de nature féodale à l'origine, avaient acquis dans la période concernée un contenu nouveau pour, dans les faits, se transformer en instruments d'accumulation primitive du capital ${ }^{20}$. Ainsi, résumait $\mathrm{A}$. Ado à la table ronde, « les études menées ces dernières décennies à propos du poids réel du prélèvement féodalo-seigneurial sur le revenu paysan dans les différentes provinces françaises, donnent de sa place dans la structure des revenus des seigneurs, de l'utilisation des terres de leur domaine un tableau beaucoup plus complexe qu'on ne se l'imaginait il y a quinze-vingt ans. » ${ }^{21}$ Et ce nouveau tableau, qui résulte des derniers acquis de l'historiographie, permet à L. Pimenova de formuler une conclusion dépourvue de toute ambiguïté : «Au niveau actuel de nos connaissances, rien ne nous autorise à définir le système des rapports sociaux existant dans la France prérévolutionnaire globalement comme un régime féodal. $»^{22}$

17. Aktual'nye problemy..., op. cit.,p. 18 , note 4.

18. Ibid., p. 94.

19. L. A. Pimenova, Dvorjanstvo nakanune Velikoj francuzskoj revoljucii (La noblesse à la veille de la Grande Révolution française), Moscou, 1986, p. 44-45.

20. A. Ado, Paysans en révolution. Terre, pouvoir et jacquerie. 1789-1794, Paris, 1996, p. 47. L'édition russe a paru en 1986.

21. Aktual'nye problemy..., op. cit., p. 10.

22. Ibid., p. 95. 
Apparemment, le premier terme de la définition utilisée par l'historiographie soviétique pour qualifier l'Ancien Régime, dit « d'absolutisme féodal », prête moins le flanc à la critique que le second, car le concept même d'absolutisme est assez largement utilisé dans la littérature de tous pays, de toutes tendances et, pour l'essentiel, personne ne le conteste. Néanmoins, les travaux les plus récents des chercheurs russes ruinent complètement la représentation de l'absolutisme propre à l'historiographie soviétique canonique de la Révolution française.

Les historiens soviétiques traitaient de l'absolutisme en France comme d'un régime politique où tout le pouvoir appartenait sans partage au monarque qui en usait avec l'arbitraire le plus complet, selon son bon vouloir. Ainsi Manfred affirmait-il : «Le roi continuait à détenir un pouvoir illimité, de nature autocratique ; c'est à lui qu'appartenait la décision finale pour les affaires intérieures et extérieures de l'État ; il nommait et destituait les ministres et les titulaires d'office ; il édictait et modifiait les lois, punissait et graciait. $»^{23}$ Revunenkov, quant à lui, écrivait : «Le roi pouvait fixer et lever tous les impôts qu'il voulait, sans demander la permission à quiconque. Il pouvait édicter et modifier toutes les lois qu'il voulait, déclarer la guerre et signer la paix, régler à sa guise toutes les affaires administratives et de justice. » ${ }^{24}$ Ainsi l'absolutisme en France était-il pratiquement identifié à l'autocratie russe, ou encore à ce que Montesquieu nommait « despotisme ».

Pourtant dans la Russie d'avant la Révolution, Pavel Ardašev, un historien qui avait passé plusieurs années en France à étudier l'Ancien Régime dans les archives, soulignait déjà que le pouvoir du roi n'était « absolu » qu'en principe, en théorie, et non dans la réalité. En pratique, il était limité par une foule de « franchises » locales et de privilèges détenus par les corporations. Même si toutes les institutions en France tenaient leurs pleins pouvoirs du roi, unique détenteur du pouvoir suprême, comme le faisait remarquer Ardašev, avec le temps ces institutions avaient gagné dans les faits une autonomie presque complète vis-à-vis de la couronne et pouvaient s'opposer à sa politique de manière très efficace ${ }^{25}$.

L'histoire des institutions en France sous l'Ancien Régime n'a pas vraiment fait l'objet d'études de la part de la science soviétique. Ignorant les travaux de P. Ardašev et des auteurs étrangers non marxistes (dits «bourgeois » en URSS), l'historiographie soviétique propose une interprétation simplifiée à l'extrême, mais idéologiquement correcte, du rôle que la monarchie jouait à la veille des événements révolutionnaires.

Actuellement les historiens russes se penchent de nouveau sur les institutions de l'absolutisme français. Dans ce sens, il faut signaler avant tout les monographies de

23. A. Z. Manfred, op. cit., p. 21.

24. V. G. Revunenkov, op. cit., p. 36.

25. P. N. Ardašev, Provincial'naja administracija vo Francii v poslednjuju poru Starogo porjadka 1774-1789 (L'administration provinciale dans la France de la fin de l'Ancien Régime, 1774-1789), Saint-Pétersbourg, 1900-1906, 2 vol. 
Nikolaj Koposov et de Vladimir Malov sur la bureaucratie d'Ancien Régime ${ }^{26}$. Bien qu'elles soient consacrées toutes deux au règne de Louis XIV, leurs conclusions concernant les particularités du fonctionnement de la monarchie française sont valables pour tout le XVIII ${ }^{\mathrm{e}}$ siècle. Ils retiennent comme une particularité déterminante auX XVII et XVIII siècles la double aspiration de la monarchie à se libérer du contrôle des institutions traditionnelles - au premier chef des parlements - et à créer un appareil d'État centralisé (une administration, donc) qui lui permette de procéder à une modernisation de la société de la manière la plus efficace et la moins douloureuse possible. Mais ces efforts ne furent pas couronnés d'un plein succès. Comme le note Nikolaj Koposov :

«Les études les plus récentes montrent que ce serait une erreur de surestimer le degré d'absolutisme atteint par la monarchie sous Louis XIV et la profondeur de la rupture avec la tradition antérieure, comme ce serait une erreur de sous-estimer la portée de la transition accomplie au XVII siècle, d'un absolutisme adolescent à un absolutisme épanoui. Jusqu'à la fin, l'absolutisme des Bourbon reste inachevé et sans doute faut-il chercher une explication à ce phénomène en partie dans la séparation demeurée incomplète de la haute administration, soutien naturel de l'absolutisme, d'avec la noblesse parlementaire. $»^{27}$

De l'avis de Malov, c'est justement l'échec des efforts déployés par les monarques pour concentrer tout le pouvoir étatique dans leurs mains non en théorie, mais dans les faits (car une part conséquente en était de facto détenue par l'appareil traditionnel des officiers* de justice), qui a déterminé par la suite la faillite de toutes les tentatives de moderniser la société « d'en haut». En témoignent non seulement les résultats fort modestes des réformes de Colbert, avance l'auteur, mais l'histoire de toutes les entreprises réformatrices initiées par le pouvoir royal au XVIII ${ }^{\mathrm{e}}$ siècle ${ }^{28}$.

Les recherches effectuées à la veille du bicentenaire de la Révolution par Irina Bergo et Elena Lebedeva, disciples d'Ado, sur l'histoire de la France prérévolutionnaire ont obligé à réviser largement cette représentation très répandue qui faisait de l'absolutisme l'obstacle principal à la modernisation de la société. Au contraire, comme le font remarquer ces auteurs, les initiatives prises en faveur d'une répartition plus juste des impôts, de la liquidation des corporations et de la liberté du commerce des grains émanaient des ministres du roi, Machaut d'Arnouville (1749), Turgot (1776), Calonne (1786), Loménie de Brienne (1787), et ne purent être appliquées du fait de l'opposition des institutions et des ordres

26. N. E. Koposov, Vysšaja bjurokratija vo Francii XVII veka (La haute administration en France au XVIr siècle), Leningrad, 1990 ; V. N. Malov, Ž. B. Kol’ber. Absoljutitskaja bjurokratija i francuzskoe obščestvo (J. B. Colbert. L'administration de l'absolutisme et la société française), Moscou, 1991.

27. N. E. Koposov, op. cit., p. 227.

* en français dans le texte.

28. V. N. Malov, op. cit., p. 206-209. 
traditionnels, opposition que le gouvernement n'était pas en état de briser ${ }^{29}$. Autrement dit, si l'absolutisme en France avait réellement disposé de ce pouvoir autocratique dont le créditaient les historiens soviétiques de la Révolution, toute une série de bouleversements majeurs réalisés pendant la période révolutionnaire auraient été, selon toute probabilité, effectués par la monarchie elle-même, plus tôt et moins douloureusement.

À la lumière des derniers ouvrages des chercheurs russes, les représentations données par la littérature historique soviétique du potentiel répressif de la monarchie française d'Ancien Régime paraissent grandement surestimées. Ainsi Manfred, à propos du règne de Louis XVI, affirmait-il : «L'arbitraire le plus complet a régné durant les années de son gouvernement. $»^{30}$ Et voici l'opinion de Revunenkov : «Quand il était en colère, le roi pouvait faire jeter en prison qui il voulait et l'y maintenir des dizaines d'années sans jugement, sans même porter à sa connaissance les chefs d'accusation : tel fut le destin du "Masque de fer", le mystérieux prisonnier de l'époque de Louis XIV. »31

Mais dans leurs publications les plus récentes, les historiens russes analysent des épisodes historiques beaucoup plus dignes de foi que l'exemple de ce « Masque de fer » à demi-mythique, et qui témoignent des possibilités assez réduites dont le roi de France disposait dans le domaine répressif. Ainsi, dans son ouvrage, Malov suit le détail du procès de Fouquet, en 1664, au cours duquel Louis XIV ne put contraindre la Chambre de justice qu'il avait lui-même nommée à prononcer la peine de mort contre l'accusé, en dépit des très fortes pressions exercées sur les juges par le gouvernement. Et L. Pimenova, dans un de ses récents ouvrages, étudie avec minutie «l'affaire du collier de la reine », en 1786, quand, malgré le souhait manifeste de Louis XVI d'obtenir la condamnation du cardinal de Rohan, le Parlement alla à l'encontre de la volonté royale en acquittant le prévenu ${ }^{32}$.

29. I. B. Bergo, «Parlamenty i političeskaja bor’ba vo Francii nakanune Velikoj francuzskoj revolucii » (Les parlements et la lutte politique en France à la veille de la Grande Révolution française), Novaja i novejšaja istoria (Histoire moderne et contemporaine), 6, 1988 ; I. B. Bergo, «Parlamentskaja oppozicija absoljutizmu i popytki reform v 1749-1776 godah » (L'opposition parlementaire à l'absolutisme et les tentatives de réformes en 1749-1776), in Francuzskaja revoljucija XVIII veka ; ekonomika, politika, ideologija (La révolution français du XVIII siècle: économie, politique, idéologie), Moscou, 1988 ; E. I. Lebedeva, «Dvorjanstvo i nalogovye privilegii nakanune revoljucii » (La noblesse et les privilèges d'imposition à la veille de la Révolution), in ibid. ; id., " Sobranija notablej kanuna Velikoj francuzskoj Revoljucii i evoljucija političeskih pozicij dvorjanstva » (Les Assemblées de notables à la veille de la Grande Révolution française et l'évolution des attitudes de la noblesse en politique), in Francuzskij Ežegodnik. 1985 (Annuaire français. 1985), Moscou, 1987.

30. A. Z. Manfred, op. cit., p. 22.

31. V. G. Revunenkov, op. cit., p. 39

32. L. A. Pimenova, «Delo ob ožerel'e Marii Antuanety » (L'Affaire du collier de MarieAntoinette), in Kazus : Individual'noe i unikal'noe v istorii. 1996 (Casus : l'individuel et l'unique dans l'histoire. 1996), Moscou, 1997, vol. 1. 
Qualifier les événements de la fin du XVIII siècle en France de «révolution bourgeoise » n'apparaît plus aussi incontestable que naguère. Conformément à la sociologie marxiste, le caractère «bourgeois » de la Révolution est déterminé avant tout par ses objectifs, qui sont « la destruction du régime féodal ou de ses survivances, l'accession au pouvoir de la bourgeoisie, ce qui crée les conditions d'un développement capitaliste ». D'autre part, les « premières révolutions bourgeoises », au nombre desquelles figure la Révolution française, sont caractérisées par le fait que « la bourgeoisie en assure la direction, et en constitue la force prépondérante $»^{33}$. Les résultats des études effectuées par les savants russes ces derniers temps non seulement ne confortent pas, mais dans une large mesure viennent contredire ces représentations.

À première vue, la participation active de la bourgeoisie aux événements de 1789-1799 ne demande pas à être démontrée. Au XVIII siècle, le terme de bourgeoisie était bien connu et était employé pour désigner les couches supérieures urbaines du Tiers-État, d'où étaient effectivement issus nombre de chefs révolutionnaires. Pourtant, comme le montre dans son étude Evgenij Kožokin, un disciple d'Ado, dans la France de l'Ancien Régime ce terme avait un tout autre sens que celui dans lequel la sociologie marxiste l'emploiera plus tard. Au XVIII siècle, constate-t-il, la bourgeoisie est essentiellement une catégorie de nature juridique et, en partie, socio-culturelle. On désignait ainsi les habitants des villes qui appartenaient au Tiers-État, avaient un statut juridique bien défini et qui se distinguaient des autres groupes sociaux par un genre de vie particulier ${ }^{34}$. Or les historiens soviétiques de la génération précédente mettaient dans ce concept un contenu avant tout socio-économique : « la classe dominante dans la société capitaliste, propriétaire des moyens de production, exploitant le travail salarié, se compose de gros, de moyens et de petits capitalistes. ${ }^{35}$ Autrement dit, il s'agit de deux concepts fondamentalement différents, regroupés sous le terme unique de «bourgeoisie ». Les historiens soviétiques de la Révolution française, en règle générale, l'utilisaient sans faire la distinction entre les deux acceptions ${ }^{36}$.

Une telle approche pourrait-elle se justifier? Peut-être ces deux définitions se rapportent-elles à un seul et même groupe social en fait, et reflètent-elles simplement des aspects différents de son existence ? Les recherches russes contemporaines montrent qu'il n'en est rien. D'abord la couche économiquement active dans la société française du XVIII ${ }^{\mathrm{e}}$ siècle ne se compose pas seulement de roturiers, mais aussi, dans une mesure non négligeable, de représentants de la noblesse et du clergé37. Et puis, insiste Kožokin, dans l'Ancien Régime, l'appartenance formelle

33. Sovetskij enciklopedičeskij slovar' (Dictionnaire encyclopédique soviétique), Moscou, 1979, p. 181 .

34. E. M. Kožokin, « Francuzskaja buržuazija na ishode Starogo porjadka » (La bourgeoisie française à la fin de l'Ancien Régime), Buržuazija iVelikaja francuzskaja revoljucija (La bourgeoisie et la Grande Révolution française), Moscou, 1989, p. 32-36.

35. Sovetskij enciklopedičeskij slovar', op. cit., p. 181.

36. A. Z. Manfred, op. cit., p. 26-28 ; V. G. Revunenkov, op. cit., p. 33.

37. L. A. Pimenova, Dvorjanstvo..., op. cit., p. 49 ; E. M. Kožokin, op. cit., p. 25-27. 
de telle ou telle personne à la «bourgeoisie» (au sens traditionnel du mot) ne suppose en rien sa participation à la vie économique. Au contraire, au milieu du XVIII ${ }^{\mathrm{e}}$ siècle, domine dans la société française une représentation du bourgeois comme d'un homme qui n'exerce pas d'activité productive, qui jouit d'une certaine aisance et vit à la noble*, d'une rente d'État ou privée ${ }^{38}$. Ainsi le concept de «bourgeoisie» utilisé dans l'interprétation canonique soviétique englobe deux groupes sociaux bien distincts. Dans ce cas, lequel d'entre eux est «le groupe hégémonique » au sein de la Révolution?

Les historiens soviétiques considèrent traditionnellement les constitutionnels qui tinrent le premier rôle dans les Assemblées nationales de 1789 à 1792 et, dès 1791, s'unirent autour du Club des Feuillants ${ }^{39}$, comme «le parti de la grande bourgeoisie ». Mais tout récemment, l'étude du statut social des membres de ce club menée par l'un des élèves d'Ado, Andrej Tyrsenko, a montré que les représentants des patrons d'industrie (c'est-à-dire « la bourgeoisie » au sens marxiste du terme) ne constituent dans ce « parti » qu'une minorité assez réduite. Ainsi, sur 601 membres dont $\mathrm{A}$. Tyrsenko est parvenu à identifier le statut, on peut en rattacher 97, c'est-à-dire pas plus de $16 \%{ }^{40}$, à la catégorie des patrons d'industrie - et ce en y mettant encore pas mal de conditions. Et cela, je le répète, alors qu'il est question $\mathrm{du}$ « parti bourgeois » de la Révolution française. Certes, même si ces faits parlent suffisamment d'eux-mêmes, cela n'autorise pas à en tirer des conclusions de longue portée. Pourtant, des recherches similaires faites à l'étranger donnent souvent des résultats analogues, ce qui suscite chez les historiens russes de sérieux doutes quant à « la situation hégémonique » de la bourgeoisie capitaliste dans la Révolution française. La disparition pratiquement totale du concept de « révolution bourgeoise » dans la littérature scientifique contemporaine en apporte indirectement la preuve.

À la lumière des recherches les plus récentes menées par les historiens russes en histoire sociale de la France, on ne peut plus, tant s'en faut, considérer comme incontestable l'une des thèses essentielles de l'historiographie soviétique, qui faisait autrefois figure d'axiome, à savoir que la Révolution française avait donné « l'impulsion la plus formidable à la formation d'un système socio-économique nouveau, le capitalisme ${ }^{41}$. Dans son étude fondamentale sur l'histoire agraire, Ado en vient à cette conclusion que la redistribution de la propriété du sol effectuée

* en français dans le texte.

38. E. M. Kožokin, ibid., p. 35.

39. A. Z. Manfred, op. cit., p. $94,97$.

40. A. V. Tyrsenko, Fel'jany (U istokov francuzskogo liberalizma) (Les Feuillants (Aux sources du libéralisme français)), Moscou, 1999, p. 106.

41. V. G. Revunenkov, op. cit., p. 505. 
sous la Révolution au profit des paysans est loin d'avoir eu un effet favorable sur le cours de la révolution industrielle dans la France du XIX ${ }^{\mathrm{e}}$ siècle :

« La division de la propriété du sol en cours dans cette période, combinée avec la conservation des institutions communautaires traditionnelles, amenait à ce que ce même paysan appauvri puisse ne pas quitter son village s'il détenait une parcelle de terre et pouvait compter sur les communaux et les droits d'usage. Cela renforçait la surpopulation agraire, entravait l'afflux des pauvres vers les villes et créait dans les villages une réserve immense de main-d'œuvre ayant absolument besoin de revenus complémentaires. Par cela même s'est prolongée dans le temps la résistance relative des formes "pré-industrielles" (artisanales et manufacturières) de la production industrielle, dont les profits étaient assurés par l'utilisation du travail bon marché des pauvres ruraux et non par la modernisation avec utilisation de machines et de technologies nouvelles. De la même façon a été freinée la modernisaton agronomique ; les traits du système traditionnel d'exploitation ont gardé partout une grande vitalité [...]. »42

L'un des principaux facteurs de ralentissement de la révolution industrielle en France est la «crise de l'investissement ». Aleksandr Reviakin montre, dans son étude des conséquences économiques de la Révolution, que la crise de l'industrie et du commerce qu'elle a provoquée a stimulé la réorientation des capitaux vers des opérations immobilières spéculatives ; ces dernières ont en effet connu un essor considérable avec la vente massive des biens nationaux. «L'augmentation sensible des investissements dans l'immobilier au détriment des investissements dans la production apparaît comme une caractéristique de la domination du capital marchand au début du XIX ${ }^{\mathrm{e}}$ siècle », écrit Reviakin.

«Ce type d'investissement improductif attirait la bourgeoisie non seulement pour la sécurité qu'il offrait, motif non négligeable dans un contexte d'instabilité politique et militaire, mais aussi du fait du prestige considérable dont jouissaient dans la société les grands propriétaires fonciers. Par ses efforts pour s'adapter à des rapports sociaux fondés sur la propriété du sol comme principal moyen de production, le capital marchand faisait la preuve de son incapacité à provoquer leur transformation radicale. $»^{43}$

En d'autres termes, la représentation trop linéaire que les historiens soviétiques de la génération précédente donnent d'un lien de cause à effet immédiat entre le développement du capitalisme en France et les événements révolutionnaires de la fin du XVIII siècle ne trouve pas sa confirmation dans l'historiographie russe contemporaine.

42. A. Ado, Paysans en révolution..., op. cit., p. 440.

43. V. Reviakin, «Buržuazija posle Francuzskoj revoljucii (pervaja polovina XIX v.)» (La bourgeoisie après la Révolution française (première moitié du XIX siècle), Buržuazija $i$ Velikaja ..., op. cit., p. 150. 
En faisant le bilan de tout ce qui vient d'être dit, on peut constater que l'évolution de l'historiographie russe de la Révolution française au cours de ces quinze dernières années s'est traduite d'abord par une érosion progressive pour aboutir à une révision complète de la base conceptuelle de l'interprétation de cet événement par les historiens soviétiques de la génération précédente. Il convient tout particulièrement de noter qu'à la différence d'autres branches de la science historique, ce « changement de jalons » radical n'a pas eu d'arrière-plan idéologique. Il a été le résultat de recherches concrètes et ne s'est pas accompagné de disputes bruyantes, ni d'un désaveu brutal des autorités scientifiques. Nullement opposé aux milieux professionnels, il s'est même produit si tranquillement qu'il est resté pratiquement ignoré des milieux périscientifiques. Dès 1988, L. Pimenova parlait d'un fossé entre le niveau des recherches contemporaines d'un côté, et la littérature de vulgarisation et les manuels de l'autre ${ }^{44}$. Il n'a fait que se creuser depuis. De nombreux vulgarisateurs professionnels et plus encore les auteurs de manuels continuent à faire de gros tirages avec les clichés dépassés de l'historiographie soviétique ${ }^{45}$. Cependant cette manière «évolutive» de «changer de jalons» sans «combats d'arrière-garde», pour reprendre une expression de Bahtin, a le mérite d'assurer une continuité à la recherche scientifique et de sauvegarder tous les acquis réellement valables légués par l'historiographie soviétique à la nouvelle historiographie russe.

Peu avant sa mort, Ado jugeait ainsi le processus en cours :

«On relève dans la littérature spécialisée un renouvellement de la problématique, sa diversification, une aspiration à une vision plus large de la Révolution française. L'image de la Révolution qui se dégage des travaux les plus récents gagne en variété, devient moins systématique, plus complexe, voire contradictoire. L'effort des historiens pour repenser certains jugements et schémas tout prêts hérités de notre passé historiographique est sensible [...] Au fond, tout ce qui a été dit nous autorise, comme je le pense, à conclure que l'historiographie soviétique de la Révolution française a cessé d'exister. La relève est assurée grâce à l'élaboration d'une historiographie, russe celle-là. Il n'y a pas solution de continuité avec le legs le plus positif de l'historiographie soviétique, mais la nouvelle historiographie appartient déjà à une nouvelle époque et elle offre un visage bien différencié. ${ }^{46}$

(traduit du russe par Hélène Rol-Tanguy)

Institut d'histoire universelle

Académie des sciences de Russie

117334 Moscou

Leninskij Prospekt, 32-a

44. Aktual' nye problemy..., op. cit.,p. 93.

45. Voir, par exemple, le manuel universitaire Novaja istorija stran Evropy i Ameriki (Histoire moderne des pays d'Europe et d'Amérique), Moscou, 1997.

46. A. V. Ado, «Pis'mo profesoru Šen Censinu» (Lettre au professeur Chen Tzensing), Vestnik MGU, série 8, Istorija, 5, 1996,p. 32. 